\title{
Fuzzy Complex Dynamical Networks and Its Synchronization
}

\author{
Nariman Mahdavi, Mohammad Bagher Menhaj, Jürgen Kurths, and Jianquan Lu
}

\begin{abstract}
In this paper, the robust synchronization problem of fuzzy complex dynamical networks is investigated. A fuzzy complex dynamical network is an extension to an uncertain complex dynamical network in which all sources of parametric uncertainties are modeled with fuzzy numbers, i.e., all nodes' dynamics are described by fuzzy differential equations (FDEs) that permit a better description of a real process occurring in the presence of inaccuracy. To resolve the synchronization problem, this paper introduces new adaptive and impulsive controllers in which globally exponential synchronization of fuzzy dynamical networks under easily verified conditions is guaranteed. Moreover, we propose an efficient method that helps to find certain suitable nodes to be impulsively controlled via pinning, noting that these nodes, in general, vary at distinct impulsive time instants. Therefore, by using adaptive controllers and applying impulsive controllers to only a small portion of nodes, the whole network will completely be synchronized to a certain objective state. Finally, two numerical examples are given to illustrate the effectiveness of the proposed controllers.
\end{abstract}

Index Terms-Adaptive control, complex dynamical networks, fuzzy differential equations (FDEs), impulsive control, pinning control, synchronization.

\section{INTRODUCTION}

A $\mathrm{N}$ INCREASING number of systems in nature, engineering, or socioeconomic systems can be modeled as networks of interconnected dynamical nodes. Typically, each node of the network is a nonlinear dynamical system interacting with the others via a topology defined on the network edges. Biological neural networks, ecosystems, social groups, the Internet, World Wide Web, or electrical power grids are only some typical examples (see [1]-[4] and references therein).

Manuscript received June 12, 2011; revised February 11, 2012 and June 13, 2012; accepted August 6, 2012. Date of publication September 14, 2012; date of current version April 16, 2013. The work of J. Kurths was supported by SUMO (EU) and ECONS (WGL). The work of J.Q. Lu was supported in part by the National Natural Science Foundation of China under Grant 61175119, by the Natural Science Foundation of Jiangsu Province of China under Grant BK2010408, by the Program for New Century Excellent Talents in University under Grant NCET-10-0329, and by the Alexander von Humboldt Foundation of Germany. This paper was recommended by Associate Editor W.-Y. Wang.

N. Mahdavi and M. B. Menhaj are with the Department of Electrical Engineering, Amirkabir University of Technology, Tehran 15914, Iran (e-mail: nariman.mahdavi@gmail.com; tmenhaj@ieee.org).

J. Kurths is with the Potsdam Institute for Climate Impact Research, D-14415 Potsdam, Germany, and with the Department of Physics, Humboldt University, 12489 Berlin, Germany (e-mail: juergen.kurths@pik-potsdam.de).

J. Q. Lu is with the Department of Mathematics, Southeast University, Nanjing 210096, China, and also with the Potsdam Institute for Climate Impact Research, D-14415 Potsdam, Germany (e-mail: jqluma@gmail.com).

Color versions of one or more of the figures in this paper are available online at http://ieeexplore.ieee.org.

Digital Object Identifier 10.1109/TSMCB.2012.2214209
One of the most interesting and significant phenomena in complex dynamical networks is synchronization among all network's dynamical nodes. In fact, synchronization is one of the most typical collective behavior and basic motions in nature [5], [6]. Specifically, adaptive rules [7]-[10], impulsive methods [11]-[16], or a combination of them [17], [18] have been used for getting synchronization in networks of coupled chaotic systems.

Most of the existing literature considered only complete synchronization when both the network structure and the coupling strength are exactly known. Moreover, the network nodes' dynamics are assumed to be exactly identical, and there is no mismatch between them. However, it should be noted that due to noise and/or uncertainties, the nodes' dynamics do not obey precise state equations, and in most practical situations, only some estimations of them are available [17]. Furthermore, exact prior knowledge about coupling structures and different weightings are usually unavailable [7]. Therefore, a method to completely synchronize the whole network in spite of these uncertainties would be necessary. To deal with unknown network couplings, numerous robust and adaptive methods have been developed [7]-[27], whereas only a few papers addressed the problem of nodes' dynamics uncertainty [10], [14], [15], [26].

All of the above papers only studied networks whose dynamics are described by ordinary differential equations (ODEs). Knowing the fact that, in the mathematical modeling of real-world phenomena, as the complexity of the system being modeled increases, we are either not able to formulate an appropriate mathematical model or the model is too complicated to be useful in practice. Moreover, knowledge about dynamical systems modeled by ODEs is often incomplete or vague [28][34]. For example, parameter values, functional relationships, or initial conditions may not be known precisely. It is therefore necessary to have some mathematical apparatus to describe vague notions of existing components of uncertainty. Furthermore, uncertainties might not be of a probabilistic type [28]. In this case, fuzzy differential equations (FDEs) become natural ways to model uncertain dynamical systems. Uncertainty in the nonlinear model of manufacture dynamics [35], biological treatment of waste water [36], dynamics of the liquid level in an oil tank [37], service composition described with business process execution language [38], turbulence dynamics [39], and atmospheric and medical cybernetics [40] were taken into consideration by using FDEs.

On the other hand, a pinning control strategy was proposed to control a complex network by controlling a small fraction of all nodes [41]-[48]. Therefore, the control action is directly exerted into these pinned nodes, and then it propagates to the 
uncontrolled nodes through the connections among the nodes. A main question in pinning control is how many and what type of nodes should be controlled to force the whole network to the desired state [44]-[46]. To deal with this problem, pinning adaptive control and adaptive tuning of the coupling strength have been proposed [47], [48]. However, in spite of a simple structure and wide use of impulsive controllers in synchronization of chaotic systems [49], [50], stabilization of hybrid neural networks [51], and their effectiveness and robustness in synchronization of complex dynamical networks [11]-[18], pinning impulsive controllers are not yet studied for the synchronization of complex dynamical networks. Moreover, to the best of our knowledge, fuzzy complex dynamical networks and impulsive or adaptive control scheme for their synchronization have not also been studied.

In the line of the above discussions, this paper further considers the issue of robust synchronization of complex dynamical networks with different parameter uncertainties, where all sources of parametric uncertainty are modeled with fuzzy numbers. After that, each node's dynamics is described by FDEs that enables us to refine the description of a real process occurring in the presence of inaccuracy, by analyzing an equation or a system of differential equations defined on a space of fuzzy sets. By utilizing the stability results for FDEs and impulsive systems established in [34] and [50], respectively, we derive sufficient conditions for the existence of adaptive and impulsive controllers under which globally exponential synchronization of fuzzy dynamical networks can be guaranteed. In addition, we propose a method for finding certain suitable nodes to be impulsively controlled via pinning, noting that these nodes vary at distinct impulsive time instants. The proposed controller needs small controlling cost and is very useful in practice.

The remaining of this paper is organized as follows. In Section II, some basic definitions and terminology are introduced, and a basic result of [34] for stability of FDEs is recalled. In Section III, the problem statements with necessary assumptions are given. Section IV studies synchronization of fuzzy complex dynamical networks and their exponential convergence based on adaptive-impulsive and adaptive-pinningimpulsive controllers. The effectiveness of the proposed methods is demonstrated through numerical simulations on a directed network and a small-world network in Section V. Conclusions and future directions are finally drawn in Section VI.

\section{BASIC CONCEPTS}

Denote by $\kappa^{n}$ the set of all nonempty compact subsets of the n-dimensional Euclidean space $R^{n}$ and by $\kappa_{C}^{n}$ the subspace of $\kappa^{n}$ consisting of nonempty convex compact sets. If $A, B \in \kappa^{n}$, the Hausdorff metric $d_{H}$ on $\kappa^{n}$ is defined by

$$
d_{H}(A, B)=\max \{\rho(A, B), \rho(B, A)\}
$$

with $\rho(A, B)=\sup _{a \in A} \inf _{b \in B}\|a-b\|$, where $\|$.$\| is a norm in$ linear spaces.
Let $D^{n}$ denote the set of upper semicontinuous normal fuzzy sets on $R^{n}$ with compact support. That is, fuzzy set $u$ belongs to $D^{n}$ if and only if the $\alpha$-level set $[u]^{\alpha}$ is a nonempty compact subset of $R^{n}$ for all $0 \leq \alpha \leq 1$. The $\alpha$-level set of $u, 0 \leq$ $\alpha \leq 1$, is defined as $[u]^{\alpha}=\left\{x \in R^{n} \mid u(x) \geq \alpha\right\}$. Clearly, for $\alpha \leq \beta$, we get $[u]^{\alpha} \supseteq[u]^{\beta}$. Element $u \in D^{n}$ is said to be fuzzy convex if

$$
u(\lambda x+(1-\lambda) y) \geq \min \{u(x), u(y)\}
$$

for all $x, y \in[u]^{0}$ and all $\lambda \in[0,1]$. It is easy to verify that if $u$ is fuzzy convex, then each $[u]^{\alpha}$ is convex. Denote by $E^{n}$ the subset of fuzzy convex elements of $D^{n}$. It is possible to define metric $d_{\infty}$ on $D^{n}\left(\right.$ or $\left.E^{n}\right)$ as

$$
d_{\infty}(u, v)=\sup _{0 \leq \alpha \leq 1} d_{H}\left([u]^{\alpha},[v]^{\alpha}\right), \quad u, v \in D^{n}\left(\text { or } \quad E^{n}\right) .
$$

Let 0 be the fuzzy singleton of $D^{n}$ (or $E^{n}$ ); for each $u \in$ $D^{n}\left(\right.$ or $\left.E^{n}\right)$, write $\|u\|=d_{\infty}(u, 0)$. All of the metric spaces $\left(\kappa^{n}, d_{H}\right),\left(R^{n},\|\|.\right),\left(D^{n}, d_{\infty}\right)$, and $\left(E^{n}, d_{\infty}\right)$ are complete. A more detailed description of these spaces can be found in [52].

Consider the following FDE:

$$
\dot{x}(t)=F(t, x(t)), \quad x(0)=X_{0} \in E^{n}
$$

where $F: R_{+} \times E^{n} \rightarrow E^{n}$. There are many attempts to define a fuzzy derivative and then study FDEs. One of the earliest was a generalization of the Hukuhara derivative of a set-valued function [32]. However, soon it appeared that the corresponding solution has a drawback of becoming fuzzier as time goes by. Consequently, this formulation cannot reflect any of the behavior of ODEs such as stability and bifurcation. To resolve the problem, Hüllermeier [28] interpreted FDEs as a family of differential inclusions at each $\alpha$-level, where $0 \leq \alpha \leq 1$, as follows:

$$
\dot{x}(t) \in[F(t, x(t))]^{\alpha}, \quad x_{\alpha}(0)=x_{0} \in X_{\alpha}:=\left[X_{0}\right]^{\alpha}
$$

where $[F(., .)]^{\alpha}: R_{+} \times R^{n} \rightarrow \kappa_{C}^{n}$. Afterward, Diamond [33] proved that the attainable solution sets $A_{\alpha}\left(X_{\alpha}, t\right), \alpha \in[0,1]$ of the family of inclusions (4) on $[0, t]$ are the level sets of fuzzy set $A\left(X_{0}, t\right) \in D^{n}$ and then extended existing results of stability and periodicity to time-dependent differential inclusions [34]. The idea was to solve these differential inclusions and using the stacking theorem of Negoita and Ralescu [53] to bunch these solutions into a fuzzy solution.

Stacking Theorem [53]: Let $\left\{Y_{\alpha} \subset R^{n}: 0 \leq \alpha \leq 1\right\}$ be a family of compact subsets satisfying the following:

- $Y_{\alpha} \in \kappa^{n}$ for all $0 \leq \alpha \leq 1$;

- $Y_{\beta} \subseteq Y_{\alpha}$ for $0 \leq \alpha \leq \beta \leq 1$;

- $Y_{\alpha}=\bigcap_{i=1}^{\infty} Y_{\alpha_{i}}$ for any nondecreasing sequence $\alpha_{i} \rightarrow \alpha$ in $[0,1]$.

Then, there is fuzzy set $u \in D^{n}$ such that $[u]^{\alpha}=Y_{\alpha}$. In particular, if $Y_{\alpha}$ is also convex, then $u \in E^{n}$. Conversely, the level sets $[u]^{\alpha}$ of any $u \in D^{n}$ satisfy these conditions, i.e., $[u]^{\alpha}$ 's become also convex if $u \in E^{n}$. 
An impulsive effect is introduced to the FDE (3) as

$$
\begin{cases}\dot{x}(t)=F(t, x(t)), & t \neq t_{k} \\ x\left(t_{k}^{+}\right)=x\left(t_{k}^{-}\right)+I\left(x\left(t_{k}^{-}\right)\right), & t=t_{k} \\ x\left(t_{0}^{+}\right)=X_{0} \in E^{n}, & k=1,2, \ldots,\end{cases}
$$

where $I: E^{n} \rightarrow E^{n}, x\left(t_{k}^{+}\right)$is the right limit of $x(t)$ at $t=t_{k}$, $x\left(t_{k}^{-}\right)$is the left limit, and for each $x \in E^{n}, \lim F(t, y)=$ $F\left(t_{k}^{+}, x\right)$ exists as $(t, y) \rightarrow\left(t_{k}^{+}, x\right)$. For future references, we need the following definitions and theorems.

Definition 1: Let $V: R_{+} \times R^{n} \rightarrow R_{+}$; then, $V$ is said to belong to class $\nu_{0}$ if [50]

1) $V$ is continuous in $\left(t_{k}, t_{k+1}\right] \times R^{n}$, and for each $x \in R^{n}$, $k=1,2, \ldots, \lim _{(t, y) \rightarrow\left(t_{k}^{+}, x\right)} V(t, y)=V\left(t_{k}^{+}, x\right)$ exists;

2) $V$ satisfies Lipschitz condition $|V(t, y)-V(t, x)| \leq$ $L\|y-x\|$, where $L \geq 0$.

Definition 2: A function $V: R_{+} \times K \rightarrow R_{+}$is a Lyapunov function of the inclusion (4) on $K \subseteq R^{n}$ if [34]

1) $V(t, x) \geq \varphi(\|x\|)$ on $K \cap\left(r B^{n}\right)$ for some $r>0$ and some continuous strictly increasing $\varphi:[0, r) \rightarrow R_{+}$. $B^{n}$ is the unit ball of $R^{n}$;

$$
\frac{\partial}{\partial t} V(t, x)+\nabla V(t, x) u \leq-W(x) \leq 0
$$

for all $t \geq t_{0}$, all $x \in K$ and every $u \in[F(t, x)]^{\alpha}$, where $W: K \rightarrow R_{+}$is continuous on $K$. If $V$ is a Lyapunov function for (4) on $\mathrm{K}$, define $E=\{x \mid W(x)=0, x \in K\}$.

Definition 3 [50]: For $(t, x) \in\left(t_{k}, t_{k+1}\right] \times R^{n}$, we define the upper right derivative of Lyapunov function $V(t, x) \in \nu_{0}$ as

$D^{+} V(t, x)=\lim _{h \rightarrow 0} \sup \frac{1}{h}[V(t+h, x+h f(t, x))-V(t, x)]$.

Definition 4 [34]: If $u \in D^{n}$ is a fuzzy set and $U$ and $W \subset$ $D^{n}$ are closed subsets of $D^{n}$, we define the distance from $W$ and Hausdorff separation, respectively, by

$$
\begin{aligned}
\rho_{*}(u, W) & =\inf _{w \in W} d_{\infty}(u, w) \\
\rho_{D}(U, W) & =\sup _{u \in U} \rho_{*}(u, W) .
\end{aligned}
$$

Lemma 1: Let $A(t) \in R^{n \times n}$ be a positive definite matrix, $B(t) \in R^{n \times n}$ be a symmetric matrix, and $\lambda_{\max } B$ denotes the maximum eigenvalue of matrix $B$. Then, for any $x \in R^{n}$ and $t \in R_{+}$, we have

$$
x^{T} B(t) x \leq \lambda_{\max }\left(A(t)^{-1} B(t)\right) \cdot x^{T} A(t) x .
$$

Recall that any crisp set $X \subset R^{n}$ is also a fuzzy set, with the membership function being the characteristic function $\chi_{X}$.

Theorem 1: Let $V$ be a Lyapunov function for (4) on $K$, and its derivative is bounded above according to (6) almost everywhere on [34, Th. 4.1]. If, for each solution $x(t)$ of (4), $W(x(t))$ is absolutely continuous, then $\rho_{D}\left(A\left(x_{0}, t\right), \chi_{E}\right) \rightarrow$ 0 as $t \rightarrow \infty$. In particular, if $V=V(x)$ does not explicitly depend on $t$, then writing $E(c)=E \cap\{x \mid V(x) \leq c\}$, $\rho_{D}\left(A\left(x_{0}, t\right), \chi_{E(c)}\right) \rightarrow 0$ for some $c>0$ as $t \rightarrow \infty$.

\section{Problem Formulation}

Consider the following uncertain complex dynamical network consisting of $N$ nonidentical coupled nodes (each of them is an $n$-dimensional dynamical system) with parametric uncertainties as well as nonlinear couplings, which is described by

$$
\underline{\dot{x}}_{i}(t)=\left(A+\Delta A_{i} \underline{x}_{i}(t)+\varphi\left(t,{ }_{i}\right)+g_{i}\left({ }_{1}, \ldots, N\right) \quad t \geq 0\right.
$$

where $i=1,2, \ldots, N,{ }_{i}=\left({ }_{i 1},{ }_{i 2}, \ldots,{ }_{i n}\right)^{T} \in R^{n}$ is the state vector of node $i, A$ is an $n \times n$ matrix, $\Delta A_{i}$ are norm-bounded parametric uncertainties, $\varphi: R_{+} \times R^{n} \rightarrow R^{n}$ is a smooth nonlinear vector function, and $g_{i}: R^{n N} \rightarrow R^{n}$ values are smooth but unknown nonlinear coupling functions.

Now, consider the case that our information about the parameters' uncertainty and/or initial condition are vague, and it is only possible to describe them with fuzzy linguistic variables, e.g., "near zero," "approximately one," or "more than two." Within a fuzzy context, these linguistic variables are usually represented by proper fuzzy numbers and could be considered as a generalization of the crisp case. Therefore, the fuzzy interpretation of (7) leads to the following fuzzy complex dynamical network described by FDEs:

$$
\dot{x}_{i}(t)=\tilde{A}_{i} x_{i}(t)+\tilde{\varphi}\left(t, x_{i}\right)+\tilde{g}_{i}\left(x_{1}, \ldots, x_{N}\right) \quad t \geq 0
$$

where fuzzy states $x_{i}=\left(x_{i 1}, x_{i 2}, \ldots, x_{i n}\right)^{T} \in E^{n}$, $i=1,2, \ldots, N, \tilde{A}_{i}$ are $n \times n$ matrices with fuzzy number elements, and $\tilde{g}_{i}, \tilde{\varphi}$ satisfy the subsequent assumptions.

Assumption 1: Al: There exist nonnegative constants $r_{i j} \geq$ $0, i$, and $j=1,2, \ldots, N$ such that $\tilde{g}_{i}: E^{n N} \rightarrow E^{n}$ satisfies the following Lipschitz condition for any $x_{j}, y_{j} \in E^{n}$ :

$$
d_{\infty}\left[\tilde{g}_{i}\left(x_{1}, \ldots, x_{N}\right), \tilde{g}_{i}\left(y_{1}, \ldots, y_{N}\right)\right] \leq \sum_{j=1}^{N} r_{i j} d_{\infty}\left(x_{j}, y_{j}\right)
$$

Moreover, for any $y \in E^{n}, \quad \tilde{g}_{i}(y, \ldots, y)=0$ to guarantee the vanishing of diffusive couplings due to complete synchronization.

Assumption 2: A2: There exist positive constants $\eta_{k}$ such that $\tilde{\varphi}: R_{+} \times E^{n} \rightarrow E^{n}$ satisfies the following Lipschitz condition for any $t \in\left(t_{k-1}, t_{k}\right]$ and $x, y \in E^{n}$ :

$$
d_{\infty}[\tilde{\varphi}(t, x), \tilde{\varphi}(t, y)] \leq \eta_{k} d_{\infty}(x, y) .
$$

It is assumed that when the network achieves synchronization, all the states of all nodes become identical, namely, $x_{1}(t)=x_{2}(t)=\cdots=x_{N}(t)=s(t)$ as $t \rightarrow \infty$, where $s(t)$ is the synchronous solution of the isolated node of the system, i.e.,

$$
\dot{s}(t)=A s(t)+\tilde{\varphi}(t, s) \quad t \geq 0
$$

Here, $s(t) \in E^{n}$ is a fuzzy singleton at each $t$ and could be a fixed point, periodic, quasi-periodic, or chaotic orbit, and $A$ is an $n \times n$ matrix with fuzzy singletons as its elements, namely, $\chi_{a}$, where $a \in R$.

The main objective of this paper is to design appropriate adaptive and impulsive controllers such that the states of the nodes $x_{i}(t)$, where $i=1,2, \ldots, N$, completely synchronize 
with the state $s(t)$ of the isolated system (11), in spite of unknown parameter uncertainties, i.e.,

$$
\lim _{t \rightarrow \infty}\left\|e_{i}(t)\right\|=0 \quad i=1,2, \ldots, N
$$

where $e_{i}(t)=x_{i}(t)-s(t)$ is the synchronization error.

For the fuzzy complex dynamical network (8), impulsive controllers $I_{i}(t)$ are designed as

$$
I_{i}(t)=\sum_{k=1}^{\infty} B_{i k} e_{i}(t) \delta\left(t-t_{k}\right), \quad i=1,2, \ldots, N
$$

where $B_{i k}$ are $n \times n$ constant (fuzzy singleton) matrices to be designed later; the impulsive time instants $t_{k}$ satisfy $0 \leq t_{0}<$ $t_{1}<t_{2}<\ldots$, with $\lim _{k \rightarrow \infty} t_{k}=\infty$; and $\delta(\cdot)$ is the Dirac impulse function. Then, the fuzzy adaptive-impulsive controlled network is given by

$$
\left\{\begin{aligned}
\dot{x}_{i}(t)= & \tilde{A}_{i} x_{i}(t)+\tilde{\varphi}\left(t, x_{i}\right) & & \\
& +\tilde{g}_{i}\left(x_{1}, \ldots, x_{N}\right)+u_{i}(t) & & t \neq t_{k} \\
x_{i}\left(t_{k}^{+}\right) & =x_{i}\left(t_{k}^{-}\right)+B_{i k} e_{i}\left(t_{k}^{-}\right), & & t=t_{k} \\
x_{i}\left(t_{0}^{+}\right)= & X_{i 0} \in E^{n}, \quad i=1,2, \ldots, N, & & k=1,2, \ldots
\end{aligned}\right.
$$

where $u_{i}(t) \in E^{n}$ are fuzzy adaptive controllers to be designed later. After that, the fuzzy error dynamical systems can be obtained as

$$
\left\{\begin{aligned}
\dot{e}_{i}(t)= & A e_{i}(t)+\tilde{\tilde{A}}_{i} x_{i}(t)+\tilde{\tilde{\varphi}}\left(t, x_{i}, s\right) & & \\
& +\tilde{\tilde{g}}_{i}(x, s)+u_{i}(t), & & t \neq t_{k} \\
e_{i}\left(t_{k}^{+}\right) & =e_{i}\left(t_{k}^{-}\right)+B_{i k} e_{i}\left(t_{k}^{-}\right), & & t=t_{k} \\
e_{i}\left(t_{0}^{+}\right) & =E_{i 0} \in E^{n}, \quad i=1,2, \ldots, N, & & k=1,2, \ldots
\end{aligned}\right.
$$

where $\tilde{\tilde{A}}_{i}=\tilde{A}_{i}-A, \tilde{\tilde{g}}_{i}(x, s)=\tilde{g}_{i}\left(x_{1}, \ldots, x_{N}\right)-\tilde{g}_{i}(s, \ldots, s)$, and $\tilde{\tilde{\varphi}}\left(t, x_{i}, s\right)=\tilde{\varphi}\left(t, x_{i}\right)-\tilde{\varphi}(t, s)$.

As an example, let $(a, b, c)_{\text {tri }}$ be a triangular fuzzy number, whose support is the compact interval $[a, c]$, and the height is equal to 1 at $b$, i.e., $\operatorname{hgt}(b)=1$. In $E^{2}$, consider $\tilde{A}$ and $A$ as follows:

$$
\begin{array}{ll}
\tilde{A} & =\left[\begin{array}{cc}
(0.8,1,1.2)_{\mathrm{tri}} & (-0.1,0,0.2)_{\mathrm{tri}} \\
\chi_{0} & (-1.1,-1,-0.9)_{\mathrm{tri}}
\end{array}\right] \\
A & =\left[\begin{array}{cc}
\chi_{1} & \chi_{0} \\
\chi_{0} & \chi_{-1}
\end{array}\right] .
\end{array}
$$

The matrix $A$ shows the desired value of the system parameters, whereas, $\tilde{A}$ reflects the uncertainty on the system parameters usually described by triangular fuzzy numbers around the desired (center) value. Therefore, $\tilde{A}_{11}, \tilde{A}_{12}$, and $\tilde{A}_{22}$, indicate approximately one, approximately zero, and approximately minus one, respectively. It also shows that there is no uncertainty on $A_{21}$. We also have

$$
\tilde{\tilde{A}}=\tilde{A}-A=\left[\begin{array}{cc}
(-0.2,0,0.2)_{\operatorname{tri}} & (-0.1,0,0.2)_{\operatorname{tri}} \\
\chi_{0} & ;(-0.1,0,0.1)_{\operatorname{tri}}
\end{array}\right]
$$

where $\tilde{\tilde{A}}$ indicates approximately zero matrix.

\section{SyNCHRONIZATION OF FUZZY COMPLEX DYNAMICAL NETWORKS}

In this section, we will propose adaptive-impulsive controllers for complete synchronization of the fuzzy complex dynamical network (8). In the first subsection, the impulsive effect will be applied to all nodes, whereas in the second subsection, the pinning impulsive control strategy will be investigated. In other words, only a small portion of nodes is selected to be controlled via the impulsive controller. These controllers will be designed based on the stability results for impulsive systems as established in [50] and the stability of FDEs [34].

The fuzzy adaptive controllers $u_{i}(t)$ are chosen as follows:

$$
u_{i}(t)=-\hat{A}_{i}(t) x_{i}(t), \quad i=1,2, \ldots, N
$$

where $\hat{A}_{i}(t)$ are the estimators of the approximately zero fuzzy matrices $\tilde{\tilde{A}}_{i}$. The update laws of these fuzzy parameters are chosen as

$$
\begin{cases}\hat{A}_{i}(t)=\left\langle\hat{a}_{j k}^{i}(t)\right\rangle, & j, k=1,2, \ldots, n \\ \dot{\hat{a}}_{j k}^{i}(t)=k_{i j k} e_{i j}(t) x_{i k}(t), & t \neq t_{k}\end{cases}
$$

where $i=1,2, \ldots, N, \hat{a}_{j k}^{i}(t) \in E^{1}$, and $k_{i j k}$ are positive constants determining the rate of adaptation. Moreover, there is no change in the value of $\hat{a}_{j k}^{i}(t)$ when $t=t_{k}$.

\section{A. Adaptive-Impulsive Method}

Theorem 2: Suppose that Assumptions A1 and A2 hold, and the adaptive controllers (16) with the update laws (17) along with impulsive controllers (13) are applied to the fuzzy complex dynamical network (8). Then, the controlled dynamical network (14) becomes globally exponentially synchronized with isolated system (11) if there exist constants $\gamma_{k}>0, \alpha_{i k} \geq$ $0, i=1,2, \ldots, N$, and $k=1,2, \ldots$, such that the following conditions are satisfied:

1) For all $t \in\left(t_{k-1}, t_{k}\right]$

$$
A^{T}+A+\left(2 \eta_{k}+\sum_{j=1}^{N} r_{i j}+\sum_{j=1}^{N} r_{j i}\right) I_{n} \leq \alpha_{i k} I_{n} .
$$

2) For all $k=1,2, \ldots$

$$
\alpha_{k}\left(t_{k}-t_{k-1}\right)+\ln \beta_{k} \leq-\gamma_{k}
$$

where $\beta_{k}=\max _{1 \leq i \leq N} \lambda_{\max }\left\{\left(I_{n}+B_{i k}\right)^{T}\left(I_{n}+B_{i k}\right)\right\}$, and $\alpha_{k}=\max _{1 \leq i \leq N} \alpha_{i k}$.

Proof: Consider the following family of differential inclusions at each $\alpha$-level, $0 \leq \alpha \leq 1$ for the fuzzy error dynamical systems (14) and the update laws (17), respectively as follows:

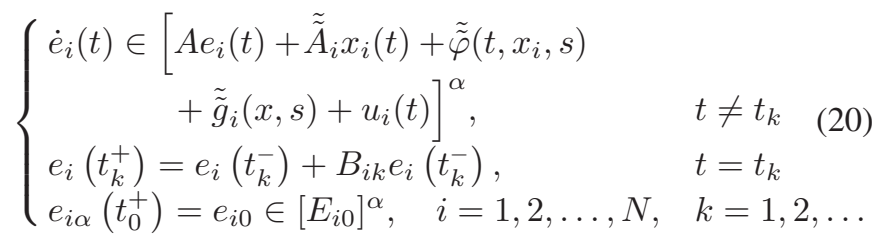

$\dot{\hat{a}}_{j k}^{i}(t) \in k_{i j k}\left[e_{i j}(t) x_{i k}(t)\right]^{\alpha}, \quad t \neq t_{k}$. 
Let $V$ be a Lyapunov function for (20) as follows:

$V_{\alpha}=\sum_{i=1}^{N} e_{i}(t)^{T} e_{i}(t)+\sum_{i=1}^{N} \sum_{j=1}^{n} \sum_{k=1}^{n} \frac{\left(\hat{a}_{j k}^{i}(t)-a_{j k}^{i}(\alpha)\right)^{2}}{k_{i j k}}$

where $\tilde{\tilde{A}}_{i}=\left\langle a_{j k}^{i}\right\rangle, a_{j k}^{i} \in E^{1}$, and $a_{j k}^{i}(\alpha) \in\left[a_{j k}^{i}\right]^{\alpha}$.

For $t \neq t_{k}$, by using the fuzzy adaptive controllers (16) with the update laws (21), we have for each $\alpha$ the following:

$$
\begin{gathered}
D^{+} V_{\alpha}=\sum_{i=1}^{N}\left(\dot{e}_{i}^{T} e_{i}+e_{i}^{T} \dot{e}_{i}\right)+2 \sum_{i=1}^{N} \sum_{j=1}^{n} \sum_{k=1}^{n} \frac{\dot{\hat{a}}_{j k}^{i}\left(\hat{a}_{j k}^{i}-a_{j k}^{i}(\alpha)\right)}{k_{i j k}} \\
=\sum_{i=1}^{N}\left[e_{i}^{T}\left(A^{T}+A\right) e_{i}+2 e_{i}^{T} \tilde{\tilde{\varphi}}^{\alpha}\left(t, x_{i}, s\right)+2 e_{i}^{T} \tilde{\tilde{g}}_{i}^{\alpha}(x, s)\right. \\
\left.\quad+2 e_{i}^{T}\left(\tilde{\tilde{A}}_{i}^{\alpha}-\hat{A}_{i}^{\alpha}(t)\right) x_{i}\right] \\
+2 \sum_{i=1}^{N} \sum_{j=1}^{n} \sum_{k=1}^{n} e_{i j} x_{i k}\left(\hat{a}_{j k}^{i}(t)-a_{j k}^{i}(\alpha)\right) .
\end{gathered}
$$

It can be easily verified that

$e_{i}^{T}\left(\tilde{\tilde{A}}_{i}^{\alpha}-\hat{A}_{i}^{\alpha}(t)\right) x_{i}=\sum_{j=1}^{n} \sum_{k=1}^{n} e_{i j} x_{i k}\left(a_{j k}^{i}(\alpha)-\hat{a}_{j k}^{i}(t)\right)$.

This leads to

$$
D^{+} V=\sum_{i=1}^{N}\left[e_{i}^{T}\left(A^{T}+A\right) e_{i}+2 e_{i}^{T} \tilde{\varphi}^{\alpha}\left(t, x_{i}, s\right)+2 e_{i}^{T} \tilde{\tilde{g}}_{i}^{\alpha}(x, s)\right] .
$$

Recall that both $\left(R^{n},\|\cdot\|\right)$ and $\left(E^{n}, d_{\infty}\right)$ are complete metric spaces, and by interpreting FDEs as a family of differential inclusions, space $E^{n}$ is changed to $\kappa_{C}^{n} \subset R^{n}$. Therefore, the Lipschitz constants in A1 and A2 remain true. Now, by using the inequality $2 x^{T} y \leq x^{T} x+y^{T} y$ that holds for any $x, y \in R^{n}$ and assumption A1, we get

$$
\begin{aligned}
\sum_{i=1}^{N} 2 e_{i}^{T} \tilde{\tilde{g}}_{i}^{\alpha}(x, s) & \leq \sum_{i=1}^{N} 2\left\|e_{i}^{T}\right\| \sum_{j=1}^{N} r_{i j}\left\|e_{j}\right\| \\
& \leq 2 \sum_{i=1}^{N} \sum_{j=1}^{N} r_{i j}\left\|e_{i}\right\|\left\|e_{j}\right\| \\
& \leq \sum_{i=1}^{N} \sum_{j=1}^{N} r_{i j}\left(e_{i}^{T} e_{i}+e_{j}^{T} e_{j}\right) \\
& =\sum_{i=1}^{N} e_{i}^{T}\left(\sum_{j=1}^{N} r_{i j}+\sum_{j=1}^{N} r_{j i}\right) e_{i}
\end{aligned}
$$

and it follows from assumption A2 that

$$
2 e_{i}^{T} \tilde{\tilde{\varphi}}^{\alpha}\left(t, x_{i}, s\right) \leq 2 \eta_{k} e_{i}^{T} e_{i} .
$$

Since the inequalities (26) and (27) hold, by replacing them in (25) and considering the first condition of Theorem 2, we obtain

$$
\begin{aligned}
D^{+} V_{\alpha} & \leq \sum_{i=1}^{N} e_{i}^{T}\left(A^{T}+A+2 \eta_{k} I_{n}+\left(\sum_{j=1}^{N} r_{i j}+\sum_{j=1}^{N} r_{j i}\right) I_{n}\right) e_{i} \\
& \leq \sum_{i=1}^{N} \alpha_{i k} e_{i}^{T} e_{i} \leq \alpha_{k} \sum_{i=1}^{N} e_{i}^{T} e_{i} \leq \alpha_{k} V_{\alpha}
\end{aligned}
$$

where $\alpha_{k}=\left(\max _{1 \leq i \leq N} \alpha_{i k}\right) \geq 0$. On the other hand, when $t=t_{k}$, the second term of the Lyapunov function does not change, and we can write

$$
\begin{aligned}
& V_{\alpha}\left(t_{k}^{+}\right)-V_{\alpha}\left(t_{k}^{-}\right) \\
& =\sum_{I=1}^{N} e_{i}^{T}\left(t_{k}^{+}\right) e_{i}\left(t_{k}^{+}\right)-\sum_{I=1}^{N} e_{i}^{T}\left(t_{k}^{-}\right) e_{i}\left(t_{k}^{-}\right) \\
& =\sum_{I=1}^{N} e_{i}^{T}\left(I_{n}+B_{i k}\right)^{T}\left(I_{n}+B_{i k}\right) e_{i}-\sum_{I=1}^{N} e_{i}^{T} e_{i} \\
& =\sum_{I=1}^{N} e_{i}^{T}\left[\left(I_{n}+B_{i k}\right)^{T}\left(I_{n}+B_{i k}\right)-I_{n}\right] e_{i}
\end{aligned}
$$

where $e_{i}=e_{i}\left(t_{k}^{-}\right)$.

From Lemma 1, we get

$$
\begin{aligned}
V_{\alpha}\left(t_{k}^{+}\right)-V_{\alpha}\left(t_{k}^{-}\right) & \leq \sum_{i=1}^{N} \lambda_{\max }\left(\left(I_{n}+B_{i k}\right)^{T}\left(I_{n}+B_{i k}\right)-I_{n}\right) e_{i}^{T} e_{i} \\
& \leq \sum_{i=1}^{N}\left(\beta_{k}-1\right) e_{i}^{T} e_{i} \leq\left(\beta_{k}-1\right) V_{\alpha}\left(t_{k}^{-}\right)
\end{aligned}
$$

where $\beta_{k}=\max _{1 \leq i \leq N} \lambda_{\max }\left(\left(I_{n}+B_{i k}\right)^{T}\left(I_{n}+B_{i k}\right)\right)$. We can also rewrite inequality (29) as $V_{\alpha}\left(t_{k}^{+}\right) \leq \beta_{k} V_{\alpha}\left(t_{k}^{-}\right)$.

Now, the globally exponential stability of the error dynamical system (20) can be obtained. By using (28), one can easily show that

$$
V_{\alpha}(t) \leq V_{\alpha}\left(t_{k-1}^{+}\right) \exp \left(\alpha_{k}\left(t-t_{k-1}\right)\right) \quad t \in\left(t_{k-1}, t_{k}\right] .
$$

Then, for $t=t_{1}^{+}$, we have

$$
V_{\alpha}\left(t_{1}^{+}\right) \leq \beta_{1} V_{\alpha}\left(t_{1}\right) \leq \beta_{1} \exp \left(\alpha_{1}\left(t_{1}-t_{0}\right)\right) V_{\alpha}\left(t_{0}\right) .
$$

Similarly, $t=t_{2}^{+}$yields that

$$
\begin{aligned}
V_{\alpha}\left(t_{2}^{+}\right) & \leq \beta_{2} V_{\alpha}\left(t_{2}\right) \leq \beta_{2} \exp \left(\alpha_{2}\left(t_{2}-t_{1}\right)\right) V_{\alpha}\left(t_{1}^{+}\right) \\
& \leq \beta_{1} \beta_{2} \exp \left(\alpha_{1}\left(t_{1}-t_{0}\right)+\alpha_{2}\left(t_{2}-t_{1}\right)\right) V_{\alpha}\left(t_{0}\right) .
\end{aligned}
$$

In general

$$
\begin{aligned}
V_{\alpha}\left(t_{k}^{+}\right) & \leq \prod_{i=1}^{k} \beta_{i} \exp \left(\sum_{i=1}^{k} \alpha_{i}\left(t_{i}-t_{i-1}\right)\right) V_{\alpha}\left(t_{0}\right) \\
& =\exp \left(\sum_{i=1}^{k} \ln \beta_{i}+\alpha_{i}\left(t_{i}-t_{i-1}\right)\right) V_{\alpha}\left(t_{0}\right) .
\end{aligned}
$$


Now, according to the second condition of Theorem 2, we get

$$
V_{\alpha}\left(t_{k}^{+}\right) \leq \exp \left(-\sum_{i=1}^{k} \gamma_{i}\right) V_{\alpha}\left(t_{0}\right)
$$

Consequently, if $\gamma_{k}>0$, where $k=1,2, \ldots$, then the fuzzy attainable set $A\left(E_{i 0}, t\right)$ converges to $\chi_{0}$ according to Theorem 1, i.e., $\rho_{D}\left(A\left(E_{i 0}, t\right), \chi_{0}\right) \rightarrow 0$ as $t \rightarrow \infty$, which means that $\left\|e_{i}(t)\right\| \rightarrow 0$ as $t \rightarrow \infty$, and the globally exponential stability of the fuzzy error dynamical system is then proved. It means that the fuzzy complex dynamical network (8) is exponentially synchronized with the isolated system (11) under the impulsive controllers $I_{i}$ and the adaptive controllers $u_{i}$. The proof is hence completed.

\section{B. Adaptive-Pining Impulsive Method}

In the previous section, when impulsive controllers are designed for the synchronization of fuzzy dynamical networks, all of the nodes should be controlled, leading to a high controlling cost. To resolve this problem, we will extend the socalled pinning control strategy to the impulsive control method. Therefore, the impulsive effect is directly exerted only into a small fraction of all nodes, and a successful synchronization of the whole network will be achieved.

Consider the following impulsive controllers:

$$
I_{i}(t)=\sum_{k=1}^{\infty} b_{k} e_{i}(t) \delta\left(t-t_{k}\right), \quad i=1,2, \ldots, l
$$

where the constants $b_{k} \in(-2,-1) \cup(-1,0)$, which means that the impulsive effects are stabilizing for the stabilization of error dynamical networks; the set of $l$ controlled nodes is selected at each time instant $t=t_{k}^{-}$by sorting all synchronization errors in a descending order and by choosing the first $l$ nodes that have higher norm values, i.e., $\left\|e_{1}(t)\right\| \geq\left\|e_{2}(t)\right\| \geq \cdots \geq$ $\left\|e_{l}(t)\right\| \geq\left\|e_{l+1}(t)\right\| \geq \cdots \geq\left\|e_{N}(t)\right\|$.

After adding the pinning impulsive controllers (32) to the fuzzy dynamical network (8), the fuzzy error dynamical network can be rewritten as (33), which is shown at the bottom of the page.

Theorem 3: Suppose that Assumptions A1 and A2 hold, and the adaptive controllers (16) with the update laws (17) along with the pinning impulsive controllers (32) are applied to the fuzzy complex dynamical network (8). Then, the fuzzy error dynamical network (33) is globally exponentially stable if there exist constants $\gamma_{k}>0, \alpha_{k} \geq 0$, and $0<\beta_{k}<1$, where $k=1,2, \ldots$, such that the following conditions are met:

1) For all $t \in\left(t_{k-1}, t_{k}\right]$

$$
A^{T}+A+\left(2 \eta_{k}+\sum_{j=1}^{N} r_{i j}+\sum_{j=1}^{N} r_{j i}\right) I_{n} \leq \alpha_{i k} I_{n} .
$$

2) For all $k=1,2, \ldots$

$$
\alpha_{k}\left(t_{k}-t_{k-1}\right)+\ln \beta_{k} \leq-\gamma_{k} .
$$

3) At each time instant $t=t_{k}^{-}$

$$
\begin{aligned}
& \beta_{k}>\left(1+b_{k}\right)^{2} \\
& \sum_{i=1}^{l_{k}}\left\|e_{i}(t)\right\|^{2} \geq \frac{1-\beta_{k}}{1-\left(1+b_{k}\right)^{2}} \sum_{i=1}^{N}\left\|e_{i}(t)\right\|^{2}
\end{aligned}
$$

where $\alpha_{k}=\max _{1 \leq i \leq N} \alpha_{i k}$.

Proof: By choosing the same Lyapunov function as the one discussed in the proof of Theorem 2 and following a similar proof approach when $t \neq t_{k}$, we get

$$
D^{+} V \leq \alpha_{k} V, \alpha_{k}=\max _{1 \leq i \leq N} \alpha_{i k}
$$

where $\alpha_{i k}$ satisfies condition (34).

Now, we want to prove that by selecting the appropriate pinning nodes according to the norm descending order, the following inequality is always guaranteed at the instant $t=t_{k}$ :

$$
V\left(t_{k}^{+}\right) \leq \beta_{k} V\left(t_{k}\right), \quad 0<\beta_{k}<1 .
$$

When $t=t_{k}$, the second term of the Lyapunov function does not change, and we can write

$$
V\left(t_{k}^{+}\right)-V\left(t_{k}^{-}\right)=\sum_{i=1}^{l}\left(1+b_{k}\right)^{2} e_{i}^{T} e_{i}+\sum_{i=l+1}^{N} e_{i}^{T} e_{i}-\sum_{i=1}^{N} e_{i}^{T} e_{i}
$$

where $e_{i}=e_{i}\left(t_{k}^{-}\right)$. Since $0<\beta_{k}<1$ and $\beta_{k}>\left(1+b_{k}\right)^{2}$, inequality (37) becomes

$$
\left(1-\left(1+b_{k}\right)^{2}\right) \sum_{i=1}^{l}\left\|e_{i}\right\|^{2} \geq\left(1-\beta_{k}\right) \sum_{i=1}^{N}\left\|e_{i}\right\|^{2}
$$

and after some simplifications, we have

$$
\left(1+b_{k}\right)^{2} \sum_{i=1}^{l}\left\|e_{i}\right\|^{2}+\sum_{i=l+1}^{N}\left\|e_{i}\right\|^{2} \leq \beta_{k} \sum_{i=1}^{N}\left\|e_{i}\right\|^{2}
$$

$$
\begin{cases}\dot{e}_{i}(t)=A e_{i}(t)+\tilde{\tilde{A}}_{i} x_{i}(t)+\tilde{\tilde{\varphi}}\left(t, x_{i}, s\right)+\tilde{\tilde{g}}_{i}(x, s)+u_{i}(t), & t \neq t_{k}, i=1,2, \ldots, N \\ e_{i}\left(t_{k}^{+}\right)=e_{i}\left(t_{k}^{-}\right)+b_{k} e_{i}\left(t_{k}^{-}\right), & t=t_{k}, i=1,2, \ldots, l \\ e_{i}\left(t_{k}^{+}\right)=e_{i}\left(t_{k}^{-}\right), & t=t_{k}, i=l+1, \ldots, N \\ e_{i}\left(t_{0}^{+}\right)=E_{i 0} \in E^{n}, & k=1,2, \ldots\end{cases}
$$


which can be rewritten as

$$
V\left(t_{k}^{+}\right)-V\left(t_{k}^{-}\right) \leq\left(\beta_{k}-1\right) \sum_{i=1}^{N}\left\|e_{i}\right\|^{2} \leq\left(\beta_{k}-1\right) V\left(t_{k}^{-}\right)
$$

and this is exactly inequality (39).

After that, the globally exponential stability of the fuzzy error dynamical system (33) can be obtained by doing the same approach discussed in the proof of Theorem 2. This completes the proof.

Remark 1: The inequality $\beta_{k}>\left(1+b_{k}\right)^{2}$ along with the norm descending order of synchronization errors ensures that the minimum number of nodes is selected at each time instant for the pinning control.

Remark 2: By an appropriate selection of $b_{k}, \beta_{k}$, and $\Delta t_{k}$, one can tune the convergence rate or the number of pinned nodes.

Remark 3: Condition (37) can be approved in two different ways: 1) fixing the number of pinned nodes $l_{k}$ and finding the appropriate impulses strength $b_{k}$; or 2) fixing the value of impulses strength $b_{k}$ and finding the appropriate number of pinned nodes $l_{k}$ at each time instant. Now, we are in the position to give an algorithm for the practical implementation of adaptive-pinning impulsive control to synchronize the complex dynamical networks. The algorithm is presented as follows and should be done at each time instant $t=t_{k}^{-}$, where $k=$ $1,2, \ldots, k_{s}$ :

1) Calculate parameter $\alpha_{k}$.

2) Select the suitable impulsive strengths $b_{k}$ and $0<\beta_{k}<$ 1 , and $\Delta t_{k}=t_{k}-t_{k-1}$ such that (35) and (36) are satisfied.

3) Sort norm values of all synchronization errors in the descending order.

4) If the inequality (37) is satisfied, then go to step (6); else, go to the next step. The initial value for the number of pinned nodes is $l_{k}=1$.

5) Increase the number of pinned nodes by one unit, i. e., $l_{k}=l_{k}+1$, and go back to step (4).

6) Apply the impulsive controller to the $l_{k}$ pinned nodes found in step (4).

7) (Stopping criterion) Repeat the above steps for the next impulsive time instant until the norm of all synchronization errors satisfy $\left\|e_{i}\left(t_{k_{s}}\right)\right\| \leq E$, with $i=1,2, \ldots, N$, where $E$ determines the closeness of errors to zero, and $k_{s}$ is the required impulsive time instants for the synchronization of the whole network.

\section{Simulation Results}

In this section, the effectiveness of the proposed method is demonstrated by two examples, in which the node dynamics is represented by a chaotic Chua circuit, and the network has a directed nearest neighbor and small-world structure. The Chua's circuit can be easily implemented by simple electronic circuits and hence widely used in complex dynamical networks analysis [3], [7], [11]-[14]. The numerical simulations are done in MATLAB environment.
Example 5.1: Consider the complex network (8) consisting of $N=10$ coupled nonidentical Chua's chaotic circuits. The equation governing each node is

$$
\dot{x}_{i}=\left(A+\tilde{\tilde{A}}_{i}\right) x_{i}+\tilde{\varphi}\left(x_{i}\right)+\tilde{g}_{i}\left(x_{1}, x_{2}, \ldots, x_{N}\right)
$$

where $i=1,2, \ldots, N, x_{i}=\left(x_{i 1}, x_{i 2}, x_{i 3}\right)^{T}$, and the matrices $A, \tilde{\tilde{A}}_{i}$, and the function $\tilde{\varphi}$ are

$$
\begin{aligned}
A & =\left[\begin{array}{ccc}
\chi_{0} & \chi_{-p} & \chi_{0} \\
\chi_{1} & \chi_{-1} & \chi_{1} \\
\chi_{0} & \chi_{q} & \chi_{0}
\end{array}\right] \\
\tilde{\tilde{A}}_{i} & =\left[\begin{array}{ccc}
\chi_{0} & \chi_{0} & \chi_{0} \\
\chi_{0} & \chi_{0} & \chi_{0} \\
\chi_{0} & (-0.2 i, 0,0.4 i)_{\operatorname{tri}} & \chi_{0}
\end{array}\right] \\
\tilde{\varphi}\left(x_{i}\right) & =\left[\begin{array}{c}
\chi_{-p} f\left(x_{i 1}\right) \\
\chi_{0} \\
\chi_{0}
\end{array}\right]
\end{aligned}
$$

where $f\left(x_{i 1}\right)=\chi_{m_{0}} x_{i 1}+\chi_{0.5\left(m_{1}-m_{0}\right)}\left(\left|x_{i 1}+\chi_{1}\right|-\mid x_{i 1}-\right.$ $\left.\chi_{1} \mid\right)$. To ensure the chaotic behavior of the system, the parameters taken from [54] are chosen as $p=0.59 / 0.12$, $q=0.59 / 0.162, m_{0}=-0.07$, and $m_{1}=1.5$.

In this example, there is only a parameter uncertainty on $q$; hence, only $\tilde{\tilde{a}}_{3,2}^{i}$ is modeled by a triangular fuzzy number around zero, but the other elements of $\tilde{\tilde{A}}_{i}$ are $\chi_{0}$. The triangular fuzzy number $(-0.2 i, 0,0.4 i)_{\text {tri }}$ shows that the uncertainty of the parameter $q$ is increased by changing $i$ from 1 to 10 . Moreover, the interaction is considered as follows:

$$
\tilde{g}_{i}\left(x_{1}, \ldots, x_{N}\right)=\left[\begin{array}{ccc}
\chi_{1} & \chi_{-2} & \chi_{1} \\
\chi_{0} & \chi_{0} & \chi_{0} \\
\chi_{-1} & \chi_{2} & \chi_{-1}
\end{array}\right]\left(\begin{array}{c}
x_{i 1} \\
x_{i+1,1} \\
x_{i+2,1}
\end{array}\right)
$$

where $i=1,2, \ldots, N-2$, and $\tilde{g}_{N-1}(x)=\tilde{g}_{N}(x)=0$. One can write (45) in the following form:

$$
\tilde{g}_{i}\left(x_{1}, \ldots, x_{N}\right)=\sum_{j=1}^{N} c_{i j} \Gamma x_{j}, \quad i=1,2, \ldots, N-2
$$

where $c_{i i}=c_{i, i+2}=\chi_{1}$ and $c_{i, i+1}=\chi_{-2}$; otherwise, $c_{i j}=$ $\chi_{0}$. Matrix $\Gamma$ is

$$
\Gamma=\left[\begin{array}{ccc}
\chi_{1} & \chi_{0} & \chi_{0} \\
\chi_{0} & \chi_{0} & \chi_{0} \\
\chi_{-1} & \chi_{0} & \chi_{0}
\end{array}\right]
$$

Furthermore, we obtain $r_{i j}=\|\Gamma\| \times\left\|c_{i j}\right\|=\sqrt{2}\left\|c_{i j}\right\|$, $\max _{i}\left(\sum_{j=1}^{N} r_{j i}\right)=4 \sqrt{2}$, and $\sum_{j=1}^{N} r_{i j}=4 \sqrt{2}$. It is also easy to verify that the function $\tilde{\varphi}$ satisfies assumption A2 with $\eta_{k}=\left|-p m_{0}\right|=0.34$.

All of the existing studies mentioned in the introduction are not able to completely synchronize the network even if a little 


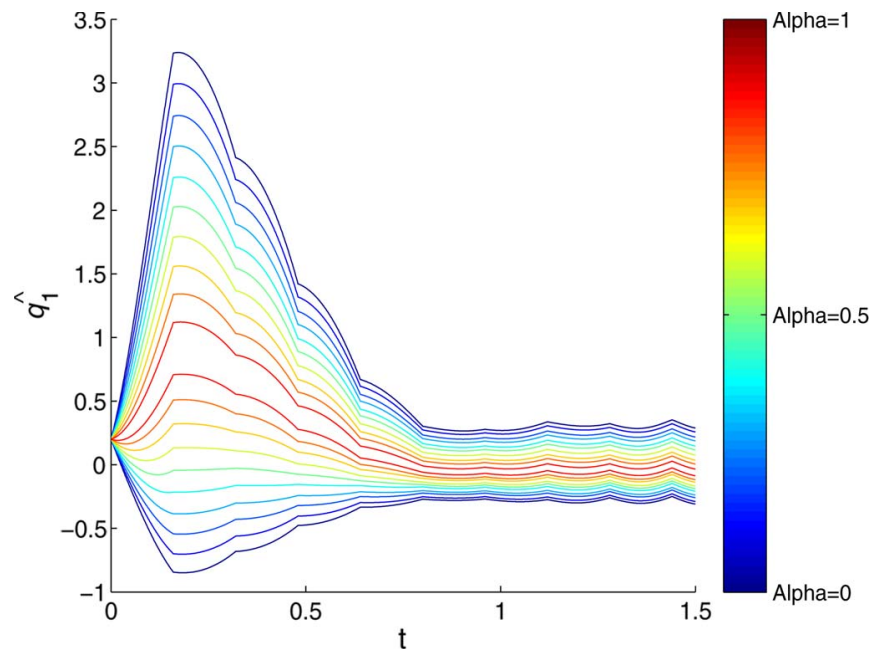

Fig. 1. Trajectory of $\hat{q}_{1}$ in the adaptive controller (46) with the update laws (47) at each $\alpha$-level, where $0 \leq \alpha \leq 1$.

amount of uncertainty exists on the dynamics of each node. Now, according to Theorem 2, for $N=10$, we get

$$
A^{T}+A+\left(2 \eta_{k}+\sum_{j=1}^{N} r_{i j}+\sum_{j=1}^{N} r_{j i}\right) I_{3} \leq \alpha_{k} I_{3}
$$

where $\quad \alpha_{k}=\lambda_{\max }\left(A^{T}+A\right)+0.68+4 \sqrt{2}+4 \sqrt{2}=17.15$ for each $\alpha$-level, where $0 \leq \alpha \leq 1$. By considering the following impulsive controllers:

$$
\begin{aligned}
t_{0} & =0, \quad t_{k}-t_{k-1}=0.16, k=1,2, \ldots \\
B_{i k} & =\operatorname{diag}(-0.9051,-0.9051,-0.9051)
\end{aligned}
$$

and using condition 2 of Theorem $2, \beta_{k}=0.009$, and the inequality $\alpha_{k}\left(t_{k}-t_{k-1}\right)+\ln \beta_{k} \leq-\gamma_{k}$ will be fulfilled by setting $\gamma_{k}=1.9$.

The adaptive controllers and the update laws are designed as

$$
\begin{aligned}
u_{i} & =-\left[\begin{array}{ccc}
\chi_{0} & \chi_{0} & \chi_{0} \\
\chi_{0} & \chi_{0} & \chi_{0} \\
\chi_{0} & \hat{q}_{i}(t) & \chi_{0}
\end{array}\right] x_{i}(t), \quad i=1,2, \ldots, N \\
\dot{\hat{q}}_{i}(t) & =50 e_{i 3}(t) x_{i 2}(t) .
\end{aligned}
$$

The initial values for $X_{i 0}, s_{0} \in E^{3}$, and $\hat{q}_{i 0} \in E^{1}$ are chosen as

$$
\begin{aligned}
X_{i 0} & =(i-0.2, i, i+0.2)_{\operatorname{tri}}\left[\begin{array}{lll}
\chi_{1} & \chi_{1} & \chi_{1}
\end{array}\right]^{T} \\
s_{0} & =\left[\begin{array}{lll}
\chi_{0.3} & \chi_{0.6} & \chi_{1}
\end{array}\right]^{T} \\
\hat{q}_{i 0} & =\chi_{0.2}, \quad i=1, \ldots, N
\end{aligned}
$$

and then, $E_{i 0} \in E^{3}$ becomes

$$
E_{i 0}=\left[\begin{array}{c}
(i-0.5, i-0.3, i-0.1)_{\operatorname{tri}} \\
(i-0.8, i-0.6, i-0.4)_{\operatorname{tri}} \\
(i-1.2, i-1, i-0.8)_{\mathrm{tri}}
\end{array}\right]^{T} .
$$

It is illustrated in Fig. 1 that the trajectory of $\hat{q}_{1}$ does converge to the triangular fuzzy number $(-0.2,0,0.4)_{\text {tri }}$. Moreover, one can observe in Fig. 2 that a combination of adaptive and impulsive controllers efficiently synchronizes the network in spite of the parameter uncertainties since all trajectories of the error system exponentially approach zero.

Remark 4: Example 5.1, without considering any parametric uncertainties, was discussed in [7], [11], and [14], where it takes approximately seven impulsive time instants to achieve synchronization. In our example, the uncertain complex fuzzy dynamical networks are synchronized within the same time and the $\alpha$-levels, where $0 \leq \alpha \leq 1$, of uncertainty in the synchronization errors are also displayed.

Example 5.2: Consider again the fuzzy complex dynamical network in Example 5.1 with $N=30$ as follows:

$\dot{x}_{i}=\left(A+\tilde{\tilde{A}}_{i}\right) x_{i}+\tilde{\varphi}\left(x_{i}\right)+\tilde{g}_{i}\left(x_{1}, x_{2}, \ldots, x_{N}\right), \quad i=1,2, \ldots, N$

but the matrix $\tilde{\tilde{A}}_{i}$ and the interaction $\tilde{g}_{i}(x)$ are

$$
\begin{aligned}
& \tilde{\tilde{A}}_{i}=\left[\begin{array}{ccc}
\chi_{0} & \chi_{0} & \chi_{0} \\
\chi_{0} & \chi_{0} & \chi_{0} \\
\chi_{0} & (-0.2-0.04 i, 0,0.4+0.08 i)_{\operatorname{tri}} & \chi_{0}
\end{array}\right] \\
& \tilde{g}_{i}\left(x_{1}, \ldots, x_{N}\right)=\sum_{j=1}^{N} c_{i j} \Gamma x_{j}
\end{aligned}
$$

where $C=\left(c_{i j}\right)_{30 \times 30}$ is the coupling configuration matrix representing the topological structure of the network, and the elements $c_{i j}$ are defined as follows. If there exists a connection between nodes $j$ and $i(i \neq j)$, then $c_{i j}=c_{j i}>0$; otherwise, $c_{i j}=c_{j i}=0$, and the diagonal elements are defined by $c_{i i}=-\sum_{j=1, j \neq i}^{N} c_{i j}$, which ensures the diffusivity condition $\sum_{j=1}^{N} c_{i j}=0$.

In this example, $C$ is the coupling matrix of a small-world network generated by using the algorithm in [5]. The initial degree of the nodes and the rewiring probability of the edges are chosen as $k=4$ and $p=0.3$, respectively. Here, we apply the adaptive-pinning impulsive method according to Theorem 3 with the impulsive constants $b_{k}=-0.9$ and $\beta_{k}=0.1$, and the impulse intervals as $t_{0}=0$ and $t_{k}-t_{k-1}=0.04$, where $k=$ $1,2, \ldots$ and then try to find an appropriate number of pinned nodes $l_{k}$ at each time instant according to the algorithm given in Remark 3. The adaptive controllers and the update laws are the same as in the previous example.

The initial values for $X_{i 0}, E_{i 0}, s_{0} \in E^{3}$, and $\hat{q}_{i 0} \in E^{1}$ are chosen as

$$
\begin{aligned}
X_{i 0} & =(0.5 i+0.3,0.5 i+0.5,0.5 i+0.7)_{\mathrm{tri}}\left[\begin{array}{lll}
\chi_{1} & \chi_{1} & \chi_{1}
\end{array}\right]^{T} \\
s_{0} & =\left[\begin{array}{lll}
\chi_{0.3} & \chi_{0.6} & \chi_{1}
\end{array}\right]^{T} \\
E_{i 0} & =\left[\begin{array}{c}
(0.5 i, 0.5 i+0.2,0.5 i+0.4)_{\mathrm{tri}} \\
(0.5 i-0.3,0.5 i-0.1,0.5 i+0.1)_{\mathrm{tri}} \\
(0.5 i-0.7,0.5 i-0.5,0.5 i-0.3)_{\mathrm{tri}}
\end{array}\right]^{T} \\
\hat{q}_{i 0} & =\chi_{0.2}, \quad i=1, \ldots, N .
\end{aligned}
$$

At the first time instant, the parameter $\alpha_{k}=5.83$ is numerically computed, and the inequality $\alpha_{k}\left(t_{k}-t_{k-1}\right)+\ln \beta_{k} \leq$ 
(a)

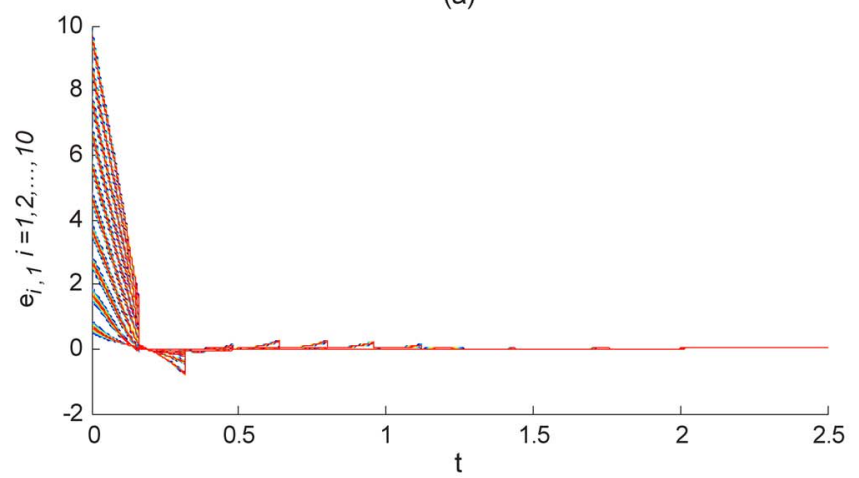

(c)

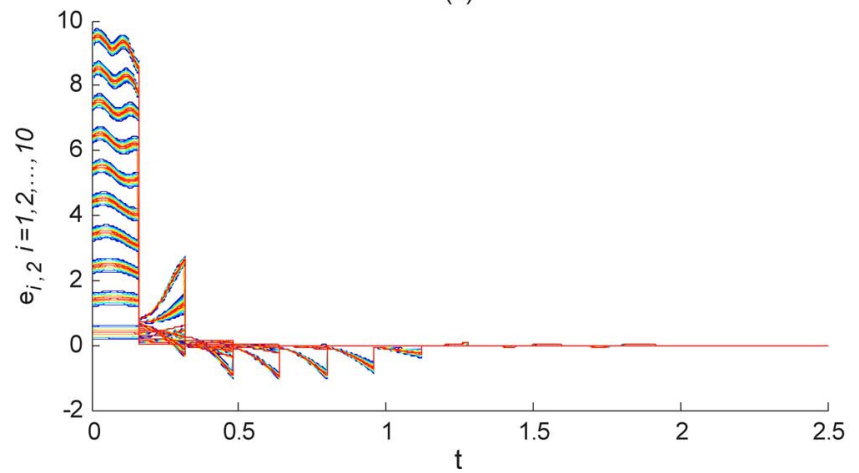

(b)

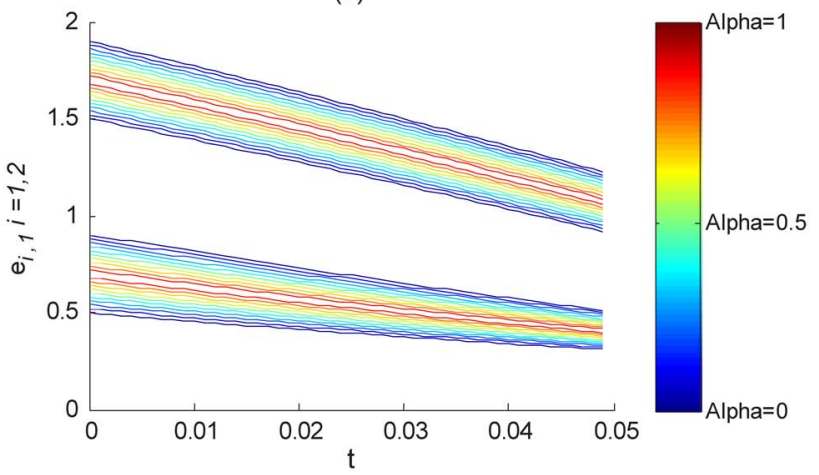

(d)

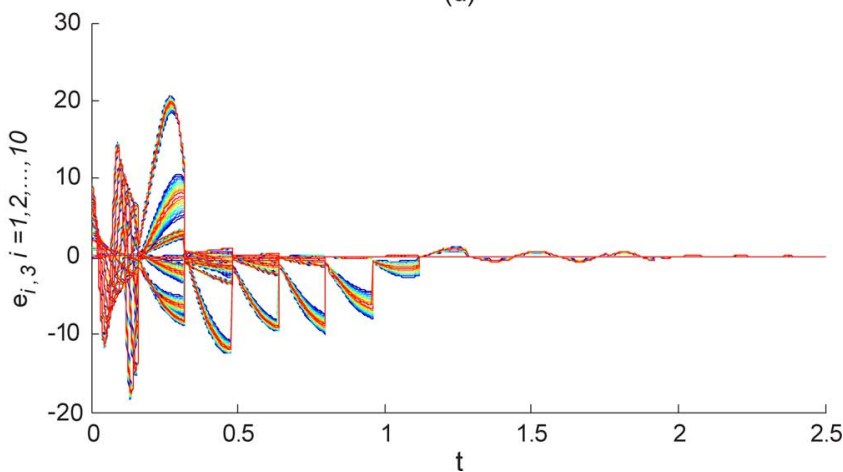

Fig. 2. Synchronization errors of fuzzy complex dynamical network (44) at each $\alpha$-level, where $0 \leq \alpha \leq 1$, by adaptive-impulsive method. (a) $e_{i 1}(t)$, where $i=1, \ldots, 10$. (b) Closer look at $e_{i 1}(t)$, where $i=1,2$. (c) $e_{i 2}(t)$, where $i=1, \ldots, 10$. (d) $e_{i 3}(t)$, where $i=1, \ldots, 10$.

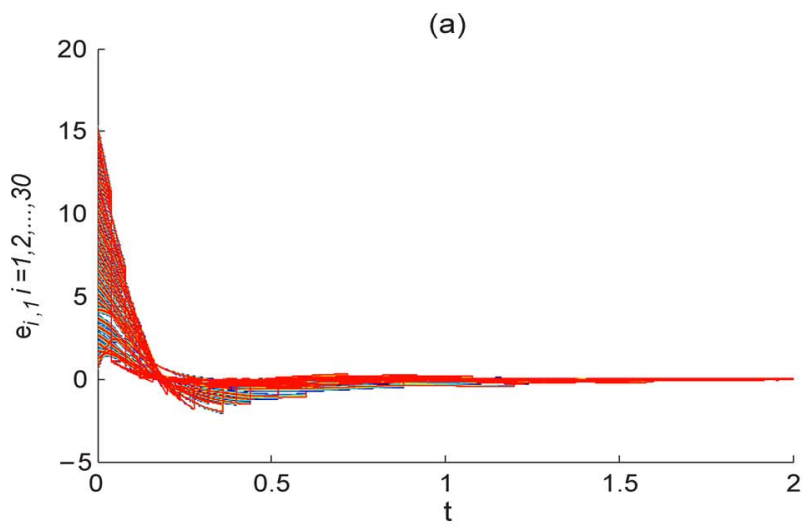

(c)

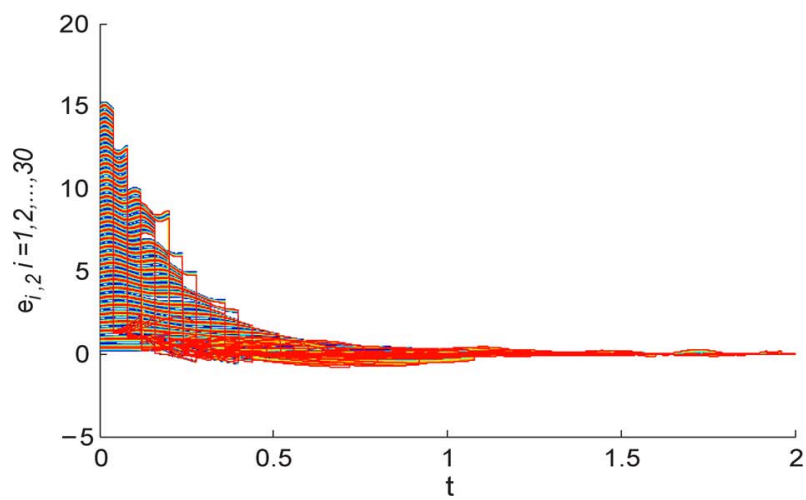

(b)

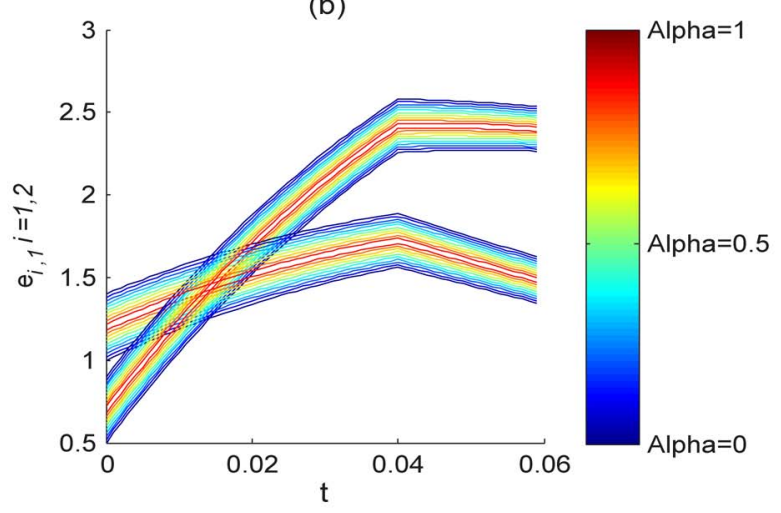

(d)

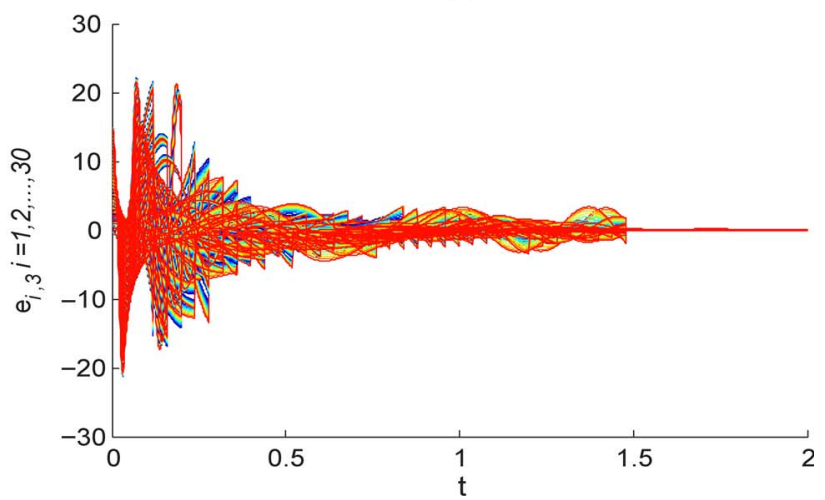

Fig. 3. Synchronization errors of fuzzy complex dynamical network (48) at each $\alpha$-level, where $0 \leq \alpha \leq 1$ by adaptive-pinning impulsive method with $\beta=0.1$. (a) $e_{i 1}(t)$, where $i=1, \ldots, 30$. (b) Closer look at $e_{i 1}(t)$, where $i=1,2$. (c) $e_{i 2}(t)$, where $i=1, \ldots, 30$. (d) $e_{i 3}(t)$, where $i=1, \ldots, 30$.

$-\gamma_{k}$ will be followed by setting $\gamma_{k}=2$. After sorting all The above procedure is repeated until the norm of all synsynchronization errors, the nodes of $27,28,26,29$, and 30 chronization errors satisfies $\left\|e_{i}\right\| \leq 0.01$, where $i=1, \ldots, N$. are selected for pinning at the first time instant, i.e., $l_{1}=5$. In this simulation, the required impulsive time instants for the 


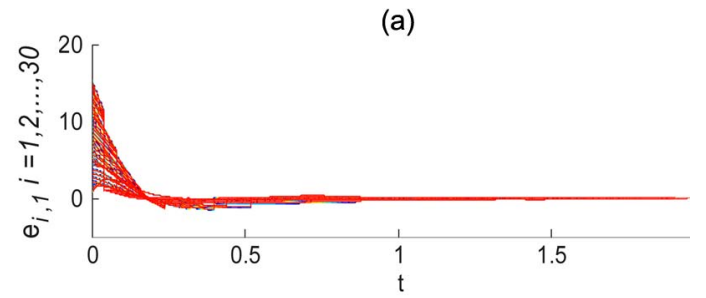

(b)

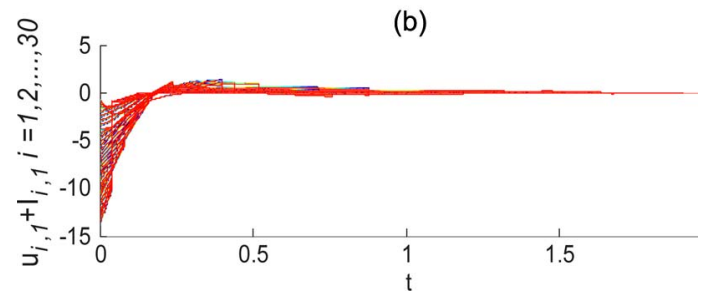

Fig. 4. (a) Synchronization errors $e_{i 1}(t)$ and (b) control inputs $u_{i 1}(t)+$ $I_{i 1}(t), i=1, \ldots, 30$, for the first state of fuzzy complex dynamical network (48) at each $\alpha$-level, where $0 \leq \alpha \leq 1$ by adaptive-pinning impulsive method with $\beta=0.05$.

synchronization of the whole network $k_{s}$ is equal to 50 , the average number of pinned nodes at each impulsive time instant $1 / k_{s} \sum_{k} l_{k}$ is equal to 4.8 , and each node is pinned eight times in average until the occurrence of synchronization, i.e., $1 / N \sum_{k} l_{k}=8$. Whereas, in the conventional impulsive controllers [11]-[18], all nodes should be pinned at each time instant; therefore, we have $1 / k_{s} \sum_{k} l_{k}=N$, and $1 / N \sum_{k} l_{k}=k_{s}$.

The synchronization errors of all nodes in the network are shown in Fig. 3. It is interesting to note that, in Fig. 3(b), the impulsive controllers are not applied on nodes 1 and 2 at the first time instant, but they are affected implicitly via the interconnections of the network; hence, their errors are reduced at $t=0.04$.

For justification of the statement given in Remark 2, the value of $\beta_{k}$ is decreased from 0.1 to 0.05 . Therefore, we have expected an increment in the value of $l_{k}$ at each time instant since the right-hand side of (37) increases. The other parameters are set as before, i.e., $b_{k}=-0.9, t_{0}=0$, and $t_{k}-t_{k-1}=0.04$, where $k=1,2, \ldots$, leading to $\gamma_{k}=2.7$ instead of 2 in the previous case. Knowing the fact that $\gamma_{k}$ determines the exponential rate of convergence, from the proof of theorem 2 , we have expected less time for the occurrence of synchronization. Simulation results are shown in Fig. 4 that illustrates the first state synchronization errors of all nodes and the trajectory of the control inputs for this state. In this case, $k_{s}=25$, and $1 / k_{s} \sum_{k} l_{k}=5$.

\section{CONClusion AND Future Work}

Synchronization of fuzzy complex dynamical networks has been studied in this paper. We have modeled all sources of parametric uncertainty in a typical complex dynamical network with fuzzy numbers; therefore, all node's dynamics are described by FDEs. After that, by using stability results for FDEs and impulsive systems, we design effective adaptive-impulsive controllers that guarantee global exponential synchronization of the fuzzy network. Moreover, adaptive-pinning impulsive controllers are designed in which impulsive effects are only applied to a small fraction of nodes. The latter controllers are more useful in practice because they need less controlling costs. Two numerical examples are given to demonstrate the effectiveness of the proposed controllers.

Although this paper is promising, there are additional sources of uncertainty that incorporate into our proposed method, such as disturbances. Consideration of both parametric and disturbance uncertainties would be an interesting future research work. Moreover, we only employed type-1 fuzzy set theory that requires the developer to describe the membership values by crisp numbers. Therefore, by taking into account the imprecision of membership functions, we may think of using type-2 fuzzy sets. Finally, what we have done in this paper is a binary stability study of fuzzy systems using Lyapunov analysis. In contrast, human-generated statements would involve degrees of stability that are articulated linguistically by terms such as "weakly stable," "more or less stable," and "strongly stable," each of them being described by some appropriate fuzzy numbers [37]. We hope that this paper provides a new framework for future research on the fuzzy stability of fuzzy systems.

\section{REFERENCES}

[1] S. H. Strogatz, "Exploring complex networks," Nature, vol. 410, no. 6825, pp. 268-276, Mar. 2001.

[2] M. E. J. Newman, "The structure and function of complex networks," SIAM Rev., vol. 45, no. 2, pp. 167-256, Jun. 2003.

[3] S. Boccaletti, V. Latora, Y. Moreno, M. Chavez, and D. U. Hwang, "Complex networks: Structure and dynamics," Phys. Rep., vol. 424, no. 4/5, pp. 175-308, Feb. 2006.

[4] A. Arenas, A. Diaz-Guilera, J. Kurths, Y. Moreno, and C. S. Zhou, "Synchronization in complex networks," Phys. Rep., vol. 469, no. 3, pp. 93 153, Dec. 2008.

[5] D. J. Watts and S. H. Strogatz, "Collective dynamics of "small-world" networks," Nature, vol. 393, no. 6684, pp. 440-442, Jun. 1998.

[6] L. M. Pecora and T. L. Carroll, "Synchronization in chaotic systems," Phys. Rev. Lett., vol. 64, no. 8, pp. 821-824, Feb. 1990.

[7] L. Wang, H. P. Dai, H. Dong, Y. H. Shen, and Y. X. Sun, "Adaptive synchronization of weighted complex dynamical networks with coupling time-varying delays," Phys. Lett. A, vol. 372, no. 20, pp. 3632-3639, May 2008.

[8] J. Zhou, J. A. Lu, and J. H. Lu, "Adaptive synchronization of an uncertain complex dynamical network," IEEE Trans. Autom. Control, vol. 51, no. 4, pp. 652-656, Apr. 2006.

[9] H. Liu, J. Chen, J. A. Lu, and M. Cao, "Generalized synchronization in complex dynamical networks via adaptive couplings," Phys. A, Stat. Mech. Appl., vol. 389, no. 8, pp. 1759-1770, Apr. 2010.

[10] Y. H. Xu, W. N. Zhou, J. A. Fang, and H. Q. Lu, "Structure identification and adaptive synchronization of uncertain general complex dynamical networks," Phys. Lett. A, vol. 374, no. 2, pp. 272-278, Dec. 2009.

[11] B. Liu, X. Z. Liu, G. R. Chen, and H. Y. Wang, "Robust impulsive synchronization of uncertain dynamical networks," IEEE Trans. Circuits Syst. I, Reg. Papers, vol. 52, no. 7, pp. 1431-1441, Jul. 2005.

[12] H. B. Jiang and Q. S. Bi, "Impulsive synchronization of networked nonlinear dynamical systems," Phys. Lett. A, vol. 374, no. 27, pp. 2723-2729, Jun. 2010.

[13] Z. H. Guan, Z. W. Liu, G. Feng, and Y. W. Wang, "Synchronization of complex dynamical networks with time-varying delays via impulsive distributed control," IEEE Trans. Circuits Syst. I, Reg. Papers, vol. 57, no. 8, pp. 2182-2195, Aug. 2010.

[14] N. M. Mazdeh, M. B. Menhaj, and H. A. Talebi, "A novel robust impulsive chaos synchronization approach for uncertain complex dynamical networks," IEICE Trans. Fundam. Electron. Commun. Comput. Sci., vol. E92.A, pp. 2499-2507, Oct. 2009.

[15] Y. W. Wang, M. Yang, H. O. Wang, and Z. H. Guan, "Robust stabilization of complex switched networks with parametric uncertainties and delays via impulsive control," IEEE Trans. Circuits Syst. I, Reg. Papers, vol. 56, no. 9, pp. 2100-2108, Sep. 2009. 
[16] J. Q. Lu, D. W. C. Ho, and J. D. Cao, "A unified synchronization criterion for impulsive dynamical networks," Automatica, vol. 46, no. 7, pp. 12151221, Jul. 2010.

[17] Z. Li and G. R. Chen, "Robust adaptive synchronization of uncertain dynamical networks," Phys. Lett. A, vol. 324, no. 2/3, pp. 166-178, Apr. 2004.

[18] K. Li and C. H. Lai, "Adaptive-impulsive synchronization of uncertain complex dynamical networks," Phys. Lett. A, vol. 372, no. 10, pp. 16011606, Mar. 2008.

[19] S. Wen, S. H. Chen, and W. L. Guo, "Adaptive global synchronization of a general complex dynamical network with non-delayed and delayed coupling," Phys. Lett. A, vol. 372, no. 42, pp. 6340-6346, Oct. 2008.

[20] J. Q. Lu and J. Cao, "Synchronization-based approach for parameters identification in delayed chaotic neural networks," Phys. A, Stat. Mech. Appl., vol. 382, no. 2, pp. 672-682, Aug. 2007.

[21] W. L. He and J. D. Cao, "Adaptive synchronization of a class of chaotic neural networks with known or unknown parameters," Phys. Lett. A, vol. 372, no. 4, pp. 408-416, Jan. 2008.

[22] Y. H. Xu, W. N. Zhou, J. A. Fang, and W. Sun, "Adaptive synchronization of the complex dynamical network with non-derivative and derivative coupling," Phys. Lett. A, vol. 374, no. 15/16, pp. 1673-1677, Apr. 2010.

[23] S. Zheng, Q. S. Bi, and G. L. Cai, "Adaptive projective synchronization in complex networks with time-varying coupling delay," Phys. Lett. A, vol. 373, no. 17, pp. 1553-1559, Apr. 2009.

[24] X. B. Lu and B. Z. Qin, "Adaptive cluster synchronization in complex dynamical networks," Phys. Lett. A, vol. 373, no. 40, pp. 3650-3658, Sep. 2009.

[25] M. Sun, C. Y. Zeng, Y. W. Tao, and L. X. Tian, "Adaptive-impulsive synchronization in drive-response networks of continuous systems and its application," Phys. Lett. A, vol. 373, no. 34, pp. 3041-3046, Aug. 2009.

[26] H. G. Zhang, T. D. Ma, G. B. Huang, and Z. L. Wang, "Robust global exponential synchronization of uncertain chaotic delayed neural networks via dual-stage impulsive control," IEEE Trans. Syst., Man, Cybern. B, Cybern., vol. 40, no. 3, pp. 831-844, Jun. 2010.

[27] P. DeLellis, M. diBernardo, and F. Garofalo, "Novel decentralized adaptive strategies for the synchronization of complex networks," Automatica, vol. 45, no. 5, pp. 1312-1318, May 2009.

[28] E. Hullermeier, "An approach to modelling and simulation of uncertain dynamical systems," Int. J. Uncertainty Fuzziness, Knowl.-Based Syst., vol. 5, no. 2, pp. 117-137, Apr. 1997.

[29] E. Hullermeier, "Numerical methods for fuzzy initial value problems," Int. J. Uncertainty Fuzziness, Knowl.-Based Syst., vol. 7, no. 5, pp. 439-461, Oct. 1999.

[30] E. Hullermeier, "Approximation of uncertain functional relationships," Fuzzy Sets Syst., vol. 101, no. 2, pp. 227-240, Jan. 1999.

[31] V. Lakshimikantham and R. N. Mohapatra, Theory of Fuzzy Differential Equations and Inclusions. London, U.K.: Taylor \& Francis, 2003.

[32] J. J. Buckley and T. Feuring, "Fuzzy differential equations," Fuzzy Sets Syst., vol. 110, no. 1, pp. 43-54, Feb. 2000.

[33] P. Diamond, "Time-dependent differential inclusions, cocycle attractors and fuzzy differential equations," IEEE Trans. Fuzzy Syst., vol. 7, no. 6, pp. 734-740, Dec. 1999.

[34] P. Diamond, "Stability and periodicity in fuzzy differential equations," IEEE Trans. Fuzzy Syst., vol. 8, no. 5, pp. 583-590, Oct. 2000.

[35] S. Turnovsky, Methods of Macroeconomic Dynamics. Cambridge, MA: MIT Press, 1995.

[36] G. Olsson and B. Newell, "Wastewater treatment systems," in Modeling, Diagnosis and Control. London, U.K.: IWA Publ., 1999.

[37] R. A. Aliev and W. Pedrycz, "Fundamentals of a fuzzy-logic-based generalized theory of stability," IEEE Trans. Syst., Man, Cybern. B, Cybern., vol. 39, no. 4, pp. 971-988, Aug. 2009.

[38] Z. Ding, H. Shen, and A. Kandel, "Performance analysis of service composition based on fuzzy differential equations," IEEE Trans. Fuzzy Syst., vol. 19, no. 1, pp. 164-178, Feb. 2011.

[39] K. K. Majumdar, "Fuzzy fractals and fuzzy turbulance," IEEE Trans. Syst., Man, Cybern. B, Cybern., vol. 34, no. 1, pp. 746-752, Feb. 2004.

[40] K. K. Majumdar and D. D. Majumdar, "Fuzzy differential inclusions in atmospheric and medical cybernetics," IEEE Trans. Syst., Man, Cybern. B, Cybern., vol. 34, no. 2, pp. 877-887, Apr. 2004.

[41] W. L. Lu, X. Li, and Z. H. Rong, "Global stabilization of complex networks with digraph topologies via a local pinning algorithm," Automatica, vol. 46, no. 1, pp. 116-121, Jan. 2010.

[42] X. F. Wang and G. R. Chen, "Pinning control of scale-free dynamical networks," Phys. A, Stat. Mech. Appl., vol. 310, no. 3/4, pp. 521-531, Jul. 2002.
[43] X. Li, X. F. Wang, and G. R. Chen, "Pinning a complex dynamical network to its equilibrium," IEEE Trans. Circuits Syst. I, Reg. Papers, vol. 51, no. 10, pp. 2074-2087, Oct. 2004.

[44] F. Sorrentino, M. di Bernardo, F. Garofalo, and G. R. Chen, "Controllability of complex networks via pinning," Phys. Rev. E, vol. 75, no. 4, pp. 046103-1-046103-6, Apr. 2007.

[45] M. Porfiri and M. di Bernardo, "Criteria for global pinning-controllability of complex networks," Automatica, vol. 44, no. 12, pp. 3100-3106, Dec. 2008.

[46] X. F. Wang, X. A. Li, and J. H. Lu, "Control and flocking of networked systems via pinning," IEEE Circuits Syst. Mag., vol. 10, no. 3, pp. 83-91, Sep. 2010.

[47] J. Zhou, J. A. Lu, and J. H. Lu, "Pinning adaptive synchronization of a general complex dynamical network," Automatica, vol. 44, no. 4, pp. 9961003, Apr. 2008.

[48] W. W. Yu, G. R. Chen, and J. H. Lue, "On pinning synchronization of complex dynamical networks," Automatica, vol. 45, no. 2, pp. 429-435, Feb. 2009.

[49] T. Yang, "Impulsive control," IEEE Trans. Autom. Control, vol. 44, no. 5, pp. 1081-1083, May 1999.

[50] T. Yang, Impulsive Control Theory. Berlin, Germany: Springer-Verlag, 2001.

[51] C. Li, G. Feng, and T. Huang, "On hybrid impulsive and switching neural networks," IEEE Trans. Syst., Man, Cybern. B, Cybern., vol. 38, no. 6, pp. 1549-1560, Dec. 2008.

[52] P. Diamond and P. Kloeden, Metric Spaces of Fuzzy Sets. Singapore: World Scientific, 1994.

[53] C. V. Negoita and D. A. Ralescu, Applications of Fuzzy Sets to System Analysis. Basel, Switzerland: Brikhauser, 1975.

[54] P. Bartissol and L. O. Chua, "The double hook," IEEE Trans. Circuits Syst., vol. 35, no. 12, pp. 1512-1522, Dec. 1988.

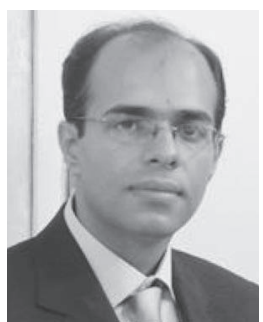

Nariman Mahdavi received the B.Sc. degree from Isfahan University of Technology, Isfahan, Iran, in 2004, and the M.Sc. (first class) and Ph.D. (first class) degrees from Amirkabir University of Technology, Tehran, Iran, in 2007 and 2011, respectively, all in electrical engineering.

From August 2010 to February 2011, he was a Guest Researcher with the Potsdam Institute for Climate Impact Research (PIK), Potsdam, Germany. His main research interests are complex systems and their synchronization, adaptive and impulsive controls, computational intelligence, and their applications in controls.

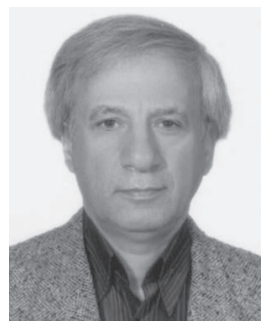

Mohammad Bagher Menhaj received the Ph.D. degree from Oklahoma State University (OSU), Stillwater, in 1992.

Then, he became a Postdoctoral Fellow with OSU. In 1993, he joined Amirkabir University of Technology, Tehran, Iran. From December 2000 to August 2003, he was with the School of Electrical and Computer Engineering, Department of Computer Science, OSU, as a Visiting Faculty Member and a Research Scholar. He has also been a Project Director for projects in the areas such as crisis control management, communication traffic control, real-time simulator design, flight control and navigation systems, and satellite attitude determination and control systems, sponsored by private and government institutions. He is author and coauthor of more than 350 technical papers, and four books: Fundamentals of Neural Networks (Amirkabir Univ. Press, Tehran 1998), Application of Computational Intelligence in Control (Amirkabir Univ. Press, Tehran 1998), Neural Networks (Amirkabir Univ. Press, Tehran 2000), and Fuzzy Computations (Danesh negar, Tehran 2007), all in Persian. He has also been a Project Director for many industrial His main research interests are: theory of computational intelligence, learning automata, adaptive filtering and their applications in control, power systems, image processing, pattern recognition, and communications, and other areas of interests are: theory of rough set and knowledge discovery. 


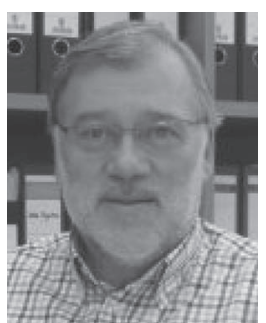

Jürgen Kurths received the B.S. degree in mathematics from the University of Rostock, Rostock, Germany; the Ph.D. degree from the Academy of Sciences of the German Democratic Republic, Berlin, Germany, in 1983; the Honorary degree from N.I. Lobachevsky State University of Nizhny Novgorod, Nizhny Novgorod, Russia, in 2008; and the Honorary degree from Saratov State University, Saratov, Russia, in 2012.

From 1994 to 2008, he was a Full Professor with the University of Potsdam, Potsdam, Germany. Since 2008, he has been a Professor of nonlinear dynamics with Humboldt University, Berlin, Germany and the Chair of the Research Domain Transdisciplinary Concepts with Potsdam Institute for Climate Impact Research, Potsdam. Since 2009, he has been the Sixth-Century Chair of Aberdeen University, Aberdeen, U.K. He is the author of more than 480 papers which are cited more than 18000 times (H-factor:56). His main research interests are synchronization, complex networks, time series analysis, and their applications

Prof. Kurths is a Fellow of the American Physical Society and a member of the Academia Europea and Macedonian Academy of Sciences and Arts. $\mathrm{He}$ is an Editor for PLoS ONE, Philosophical Transaction of the Royal Society A, CHAOS, etc. He was a recipient of the Alexander von Humboldt Research Award from the Council of Scientific and International Research (India).

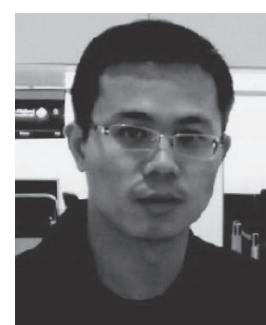

Jianquan Lu received the B.S. degree in mathematics from Zhejiang Normal University, Jinhua, China, in 2003; the M.S. degree in mathematics from Southeast University, Nanjing, China, in 2006; and the Ph.D. degree from City University of Hong Kong, Hong Kong, in 2009.

$\mathrm{He}$ is currently an Associate Professor with the Department of Mathematics, Southeast University. His current research interests include collective behavior in complex dynamical networks, multiagent systems, hybrid systems, synchronization, nonlinear systems and control, and neural networks.

Dr. Lu is an Associate Editor for Neural Processing Letters (Springer) and Neural Computing and Applications (Springer) and a Guest Editor for Mathematics and Computers in Simulation (Elsevier). He was a recipient of Jiangsu Provincial Outstanding Master's Thesis Award in 2007, an Alexander von Humboldt Fellowship in 2010, Program for New Century Excellent Talents in University by The Ministry of Education, China in 2010, and The First Award of Jiangsu Provincial Progress in Science and Technology in 2010 as the Second Project Member. 